\title{
Percepciones de las herramientas web en los entornos inclusivos
}

\author{
Begoña E. Sampedro Requena ${ }^{1}$ \\ Universidad de Córdoba \\ Juan M. Muñoz González² \\ Universidad de Córdoba
}

\begin{abstract}
Resumen: La educación inclusiva es hoy un movimiento que va cobrando una gran relevancia en todos los estados, así encontramos numerosas propuestas de investigación y de experiencias de innovación docente que denotan como la visión que se tiene sobre este movimiento ha ido evolución. Dicho crecimiento ha venido de la mano, en los últimos tiempos, del desarrollo tecnológico que la sociedad ha experimentado. De este modo, los entornos inclusivos se están viendo cada vez más afectados por su presencia. Sin embargo la valoración que de este binomio se tenga, va a venir determinada por la formación previa que se haya recibido. En este trabajo se presentan los resultados obtenidos de un grupo de maestros en formación del Grado de Educación Primaria sobre la presencia de las herramientas Web 2.0 en los entornos inclusivos. El principal resultado alcanzado refleja como los maestros generalistas del grado son los que tienen una percepción más positiva de estas en ese entorno.

Palabras clave: educación inclusiva, herramientas Web, formación, maestro en formación.
\end{abstract}

Abstract: Inclusive education is today a movement that gaining great relevance in all states. Thus we find many proposals for researches and teaching innovation experiences that indicate how the vision that has this movement has been progress. This growth has gone hand in hand, in recent times, technology development that society has experienced. In this way, inclusive environment are being increasingly affected by their presence. However, the valuation that this binomial is taken, will be determined by previous training has been received. In this paper we presents the results of a group pre service teacher in Grade of Primary Education on the presence of Web 2.0 tools in inclusive environments are presented. The principal aims achieved reflects as generalists grade teachers are those who have a more positive perception of these in that environment.

Keywords: inclusive education, web tools, trainning, teacher in pre service.

\section{INTRODUCCIÓN}

El proyecto propuesto por Casanova y González (2010) unifica la educación inclusiva y las TIC, el mismo recoge la importancia de las tecnologías de la

\footnotetext{
${ }^{1}$ Profesora de la Universidad de Córdoba, acreditada por ANECA a las figuras de Contratado Dr. y Ayudante Dr. desempeñando sus funciones docentes e investigadoras en el área de Didáctica y Organización Escolar de la Universidad de Córdoba. Doctora en Educación por la Universidad de Córdoba, Máster de Postgrado en Educación Inclusiva. Licenciada en Psicopedagogía y diplomada en Magisterio (Universidad de Córdoba). Su línea docente e investigadora se relaciona con la tecnología educativa dirigida a la formación de futuros docentes y educadores sociales, su uso y aplicabilidad para la mejora de los contextos educativos, las cuales se observan en las diferentes publicaciones, aportaciones a congresos y participación en proyectos de investigación e innovación

${ }^{2}$ Profesor Ayudante Doctor, ha participado en congresos, tanto de carácter nacional como internacional, vinculados a la Tecnología Educativa. Autor de libros y capítulos de libros relacionados con la investigación y la innovación digital publicados en editoriales como Síntesis, Paraninfo, Da Vinci, etc. Autor de artículos publicados en revistas nacionales como TESI, Teoría de la Educación. Educación y Cultura en la Sociedad de la Información, Digital Educational Review, Revista Complutense de Educación, Revista de Ciencias de la Educación, etc.; e internacionales como MAGIS: Revista Internacional de Investigación en Educación o EDUWEB: Revista de Tecnología de Información y Comunicación en Educación
} 
información y la comunicación en el desarrollo de nuevas formas de inclusión digital de la ciudadanía en la sociedad del conocimiento. Sin embargo, estos autores si abogan por comunidades virtuales para el desarrollo de la diversidad como eje de la educación inclusiva.

De todas formas, como hemos expresado con anterioridad, creemos que unas y otras se pueden beneficiar de las herramientas Web 2.0 para el desarrollo del aprendizaje en las escuelas inclusivas, en este sentido el potencial de la Web 2.0 reside en su accesibilidad a los sistemas, tanto de comunicación como de formación, favoreciendo de esta forma la participación de todos y todas, así como, sus potencialidades.

Las tecnologías de la información y la comunicación, en la actualidad las herramientas Web 2.0, pueden y tienen mucho que aportar en los entornos inclusivos, por esta razón, creemos necesario conocer si la valoración de los futuros maestros y maestras coincide con estas manifestaciones, intentando averiguar si el empleo de las tecnologías educativas Web 2.0 reforzarán o fomentaran las estrategias de desarrollo de los entornos inclusivos, presencia, participación y progreso. En esta línea, Cabero y Córdoba (2009) señalan que el uso de las herramientas Web 2.0 en las escuelas inclusivas propicia el acceso de forma más relevante a la escuela, favorecen la autonomía y la iniciativa del alumnado, y desarrolla las potencialidades de los estudiantes, dado que se convierten en motor de motivación para el aprendizaje, es decir, que estos autores consideran que el uso de las herramientas Web 2.0 en los entornos inclusivos desarrolla la presencia, la participación y el progreso, puesto que tienen las características propias y definitorias de estas estrategias inclusivas.

En resumen, debemos averiguar a través de un estudio si los futuros docentes opinan que su conocimiento y uso de las herramientas Web 2.0 en el ámbito educativo, y su aplicación a los procesos educativos reforzará o favorecerá las estrategias de presencia, participación y progreso como elementos definitorios y estratégicos de la educación inclusiva, la cual implica una educación de calidad para todos.

\section{MÉTODO}

En este estudio se ha utilizado un método de investigación de carácter no experimental y descriptivo, tal y como apunta Mateo (2004). Consecuentemente, no queremos manipular las variables independientes debido a que buscamos una relación entre las variables dependientes e independientes a posteriori, ya que el estudio se encuadra dentro del tipo ex post facto con carácter retrospectivo, como señala León (2003). Este hecho sugiere un grupo y múltiples medidas, dado que la muestra será lo más heterogénea posible, estudiando las variables dependientes en primer lugar. Esta elección, como apuntábamos con anterioridad, concibe que las hipótesis de investigación planteadas se realicen en término de cuestiones y que el análisis, así como su estudio, es decir, las relaciones que se entablen entre las distintas variables, deban ser buscadas a posteriori.

La tabla 1 muestra la distribución de la muestra en función de la mención estudiada dentro del Grado de Educación Primaria de la Facultad de Ciencias de la Educación de la Universidad de Córdoba.

Tabla 1. Distribución de la muestra 


\begin{tabular}{|c|c|c|}
\hline Mención & Frecuencia (f.) & Porcentaje (\%) \\
\hline Mención Educación Primaria Generalista & 65 & $58.56 \%$ \\
\hline Mención Educación Física & 54 & $80.60 \%$ \\
\hline Mención Lengua Extranjera & 65 & $77.38 \%$ \\
\hline Mención Educación Musical & 43 & $76.78 \%$ \\
\hline Mención Necesidades Educativas Especificas & 77 & $91.67 \%$ \\
\hline Total & 304 & $76.17 \%$ \\
\hline
\end{tabular}

Como reflejan los datos de la tabla 1, todas las menciones superan el $50 \%$ de la población a la que se adscriben, evidenciado en los cálculos anteriores, donde el valor de la muestra de nuestro estudio debe ser como mínimo 196 y los valores alcanzados son 304, lo que supone que es una muestra representativa, siendo el margen de error de $2.7 \%$. La muestra asciende a un total de 304 participantes, cuya característica descriptiva general es ser maestros y maestras de cuarto curso de la Facultad de Ciencias de la Educación de la Universidad de Córdoba para el Grado de Educación Primaria. Atendiendo al sexo comprobamos que el $70.7 \%$ de la muestra participante en la investigación pertenece al sexo femenino mientras que el $29.3 \%$ es del sexo masculino. La edad media de los alumnos y las alumnas participantes en esta investigación es de 22.48 años, siendo la edad mínima de 20 y la máxima de 47 años.

En relación a la mención estudiada, la mayoría de los alumnos y las alumnas participantes se encuentran cursando las menciones de Educación Primaria Generalista, Lengua Extranjera y Necesidades Educativas Específicas con un 21.4\%, $21.4 \%$ y $25.3 \%$ respectivamente, mientras que las menos representadas son Educación Física con un $17.8 \%$ y Educación Musical con un 14.1\%. Estos datos son representativos de la población de origen.

$\mathrm{Si}$ contrastamos las menciones en las que se encuentra matriculado el alumnado en función del sexo, como muestra la figura 1, podemos comprobar que existen diferencias entre ellas (ji cuadrado=114.266, p<0.01). En la Mención de Educación Primaria Generalista existe un mayor número de mujeres matriculadas, 84.6\%, frente a un $15.4 \%$ de hombres, mientras que en la Mención de Educación Física está circunstancia varía, ya que prevalece un mayor número de alumnado masculino (87\%) frente a un menor número de alumnado femenino (13\%). En las menciones de Lengua Extranjera y Necesidades Educativas Específicas los resultados son parecidos, dado que en ambas, el número de alumnas matriculadas es mayor que el número de alumnos, en la primera especialidad el $12.3 \%$ son hombres frente al $87.7 \%$ que son mujeres, y en la segunda el $11.7 \%$ son alumnos frente a un $88.3 \%$ que son alumnas. Por último, en la Mención de Educación Musical, el porcentaje de alumnado masculino es menor que el correspondiente al alumnado femenino (34.9\% y $65.1 \%$ respectivamente).

Con respecto a la procedencia de los alumnos y las alumnas participantes en este estudio, un poco más de la mitad son de ciudad, el 53\%, mientras que el $47 \%$ del alumnado procede del ámbito rural. En relación al tipo de vivienda donde reside la muestra, podemos observar que la residencia de los alumnos y las alumnas con mayor porcentaje es el domicilio familiar, el $64.5 \%$, el alumnado que vive fuera de la vivienda de sus padres y madres, el $35.2 \%$ prefiere residir mayoritariamente en piso de estudiantes frente al $0.3 \%$ que vive en residencia o colegios mayores.

En lo que se refiere a la disponibilidad del ordenador de los alumnos y las alumnas participantes en este estudio. Como podemos observar, más de la mitad, el $63.8 \%$, disponen de su propio ordenador frente a un $0.3 \%$ que no disponen de él.. El porcentaje del alumnado que decide disponer de un ordenador público es del $4.3 \%$ 
mientras que el $31.6 \%$ de los alumnos y las alumnas optan por disponer de ordenadores personales y públicos.

En relación al acceso a Internet que emplean los alumnos y las alumnas participantes en el estudio, podemos observar que la modalidad inalámbrica o wifi es la más utilizada (29.9\%). El $23.4 \%$ utiliza la tipología familiar, es decir, un acceso a través del servicio contratado por las familias, mientras que la modalidad pública sólo la utilizan un $10.2 \%$ y, el empleo a través del móvil es de $7.9 \%$. Todas estas tipologías de acceso (familiar, pública, wifi y móvil) son utilizadas simultáneamente por el $27.3 \%$, en contra, sólo el $1.3 \%$ de la muestra no accede a Internet a través de ninguna modalidad.

Si comparamos las modalidades de acceso a Internet que emplea el alumnado con las titulaciones en las que se encuentra matriculado, podemos comprobar que existen diferencias entre ellas (ji cuadrado $=53.069, \mathrm{p}=0.033$ ). En relación a la tipología de acceso familiar, los alumnos y las alumnas que más la utilizan son los de Educación Especial (31\%) mientras que los y las que menos la usan son los de Educación Musical (8.5\%), las titulaciones de Educación Física y Lengua Extranjera emplean por igual el acceso a Internet familiar (16.9\%) y Educación Primaria Generalista la utiliza un $26.8 \%$.

El acceso a Internet de tipo público es mayoritariamente utilizado por la titulación de Lengua Extranjera (41.9\%) en cambio el alumnado matriculado en Educación Física es quien menos lo utiliza (6.5\%), las titulaciones de Educación Primaria Generalista y Necesidades Educativas Específicas lo utilizan un 22.6\% y un $19.4 \%$ respectivamente. Los alumnos y las alumnas de Educación Musical emplean el acceso público un $9.7 \%$.

Respecto al acceso a Internet a través de la modalidad inalámbrica o wifi, observamos que el alumnado matriculado en todas las titulaciones presentan valores próximos, aunque Educación Especial lo emplea con un $28.6 \%$ mientras que Educación Física sólo un 14.3\%. Las menciones de Lengua Extranjera, Educación Primaria Generalista y Educación Musical poseen valores de $16.5 \%, 18.7 \%$ y $22 \%$ respectivamente.

En relación a la modalidad de acceso móvil, los estudiantes que más la utilizan son los de Lengua Extranjera (33.3\%) mientras que los y las que menos la usan son los de Educación Musical (8.3\%). Las titulaciones de Educación Primaria Generalista y Necesidades Educativas Específicas emplean de manera parecida el acceso a Internet a través del móvil (16.7\% y $12.5 \%$ respectivamente), en cambio, la especialidad de Educación Física la utiliza un 29.2\%.

$\mathrm{El}$ acceso a Internet a través de todas las modalidades (familiar, público, wifi y móvil) es mayoritariamente utilizado por la titulación de Educación Física (24.1\%), en cambio, el alumnado matriculado en Educación Musical es quien menos lo utiliza (12\%); las titulaciones de Educación Primaria Generalista y Lengua Extranjera lo utilizan por igual el acceso a través de todas las tipologías (20.5\%), mientras que los alumnos y las alumnas de Educación Especial lo emplean un 22.9\%.

Por último, podemos observar que de los 4 estudiantes que no acceden a Internet, no existe alumnado matriculado en las titulaciones de Educación Física y Lengua Extranjera, en cambio, no acceden Internet algunos de las titulaciones de Educación Primaria Generalista, Necesidades Educativas Específicas y Educación Musical (25\%, 25\% y 50\% respectivamente).

En resumen, podemos decir que el acceso a través de las modalidades familiar, inalámbrica o wifi y el uso de todas las tipologías (familiar, público, wifi y móvil) son 
las formas más empleadas para acceder a Internet en el conjunto de todas las titulaciones mientras que las otras formas de acceso tienen menor frecuencia.

Contrastando las modalidades de acceso a Internet que emplea el alumnado con el tipo de vivienda donde reside, podemos comprobar que existen diferencias entre ellas (ji cuadrado $=117.254, \mathrm{p}<0.01$ ).. En relación a la tipología de acceso familiar, los alumnos y las alumnas que más la utilizan son los que viven en el domicilio familiar (90.1\%). mientras que los y las que menos la usan son los y las que residen en piso de estudiantes (9.9\%). Por el contrario, el alumnado que vive en un colegio mayor o residencia no utiliza esta modalidad de acceso.

El acceso a Internet de tipo público es mayoritariamente utilizado por el alumnado que vive en su domicilio familiar (64.5\%), en cambio, el residente en piso de estudiantes lo emplea un 35.5\%. Respecto al acceso a Internet a través de la modalidad inalámbrica o wifi, observamos que más de la mitad del alumnado que vive en piso de estudiantes lo utiliza (56\%), mientras que un $44 \%$ corresponde a los alumnos y las alumnas que residen en domicilio familiar. En relación a la modalidad de acceso móvil, los alumnos y las alumnas que más la utilizan son los que viven en el domicilio familiar (70.8\%) mientras que los y las que menos la usan son los de piso de estudiantes. El acceso a Internet a través de todas las modalidades (familiar, público, wifi y móvil) es mayoritariamente utilizado por el alumnado que vive en el domicilio familiar (66.3\%) en cambio el que reside en piso de estudiantes lo emplea en menor cuantía (33.7\%). Por último, podemos observar, que no existe alumnado que viva en domicilio familiar que no acceda, en cambio no acceden a Internet los que residen en un colegio mayor o residencia y en piso de estudiantes ( $25 \%$ y $75 \%$ respectivamente).

En resumen, podemos decir que los alumnos y alumnas que viven en un colegio mayor o residencia no acceden a Internet, mientras que los que residen en domicilio familiar utilizan mayoritariamente el acceso de modalidad familiar y los de piso de estudiantes el acceso inalámbrico o wifi. Por esta razón, para la elaboración del instrumento hemos considerado los criterios (ver tabla 2) propuestos por Pérez, García, Gil y Galán (2009, pp.36-37) para la realización de un cuestionario que ostente los máximos parámetros de validez y fiabilidad, así como, que posea rigor científico.

Tabla 2. Criterios fundamentales para la elaboración de un instrumento con rigor científico. Fuente: Pérez et al. (2009).

\section{CRITERIOS}

a) Identificación y definición clara y concisa del rasgo o característica

b) Conocer los objetivos a conseguir con el instrumento

c) Seleccionar los ítems o elementos más apropiados

d) Formular los ítems elementos con precisión

\section{DESCRIPCIÓN}

Los elementos deben recoger aspectos relevantes y operativos, con definiciones claras y estructuradas en categorías evaluables.

Los objetivos deben ser trasladados a los elementos o ítems que configuran el instrumento, los cuales deben considerar el grado de dominio del sujeto y la redacción de los mismos.

Para esta acción se deben considerar las características de los sujetos a los que va dirigido el instrumento y la dificultad, homogeneidad y validez de los ítems.

Se debe buscar la facilidad en la comprensión de los

o ítems o elementos que configuran el cuestionario, considerando el vocabulario elegido, así como, la claridad de los mismos. 
Considerar los criterios de dificultad (preferiblemente de menor a mayor), combinando

e) Ordenar los ítems de los tipos de elementos o ítems con una secuencia forma apropiada.

lógica. Es preferible realizar instrucciones por bloques o dimensiones en las que se presenten los ítems.

Se debe tener en cuenta las características de los sujetos a los que va dirigido el instrumento, considerando el momento de la aplicación y la

f) El tiempo preciso para responder (duración) finalidad de los resultados. Así mismo, se debe calcular la temporalización de la cumplimentación de la prueba o instrumento, tanto de forma conjunta como por partes o dimensiones.

g) Las respuestas que debe Para este criterio, se considera el tipo de respuestas dar el sujeto

las cuales deben ser univocas y registrarse con facilidad, de esta forma se evitarán errores de interpretación.

En un primer lugar, y teniendo en cuenta los criterios a), b) y c), consultamos distinta documentación sobre las herramientas Web 2.0, definición, origen, aplicación, utilidad para la escuela, clasificación, etc., dado que todos los autores revisados coincidían en que existen más de 3500 tipos de herramientas (Marín y Reche, 2011).

Considerando que el problema planteado se concretaba en una etapa educativa, de forma concreta, decidimos seleccionar las herramientas Web 2.0 más utilizadas en la etapa de primaria; por esta razón, nos consideramos la tipología que realiza Castaño et.al. (2008, pp.64-65) para clasificar las herramientas Web 2.0, asegurándonos que las tres categorizaciones, aplicaciones on-line, herramientas de publicación y gestión de la información, estuvieran representadas en nuestro instrumento.

En un segundo momento, y considerando que el tema que queríamos tratar recogía dos temáticas muy ilimitadas, como son, las características o elementos descriptivos que propone Booth y Dyson (2006, citado en Muntaner, 2010, pp. 9) sobre la práctica de la educación inclusiva, presencia, participación y progreso, y las ocho herramientas Web 2.0 seleccionadas, reflexionamos que no podíamos diseñar ítems o elementos para el instrumento que tuvieran carácter de afirmación, interrogación o declaración. Por este motivo, planteamos presentar cada una de las ocho herramientas para su medición en categorías. Finalmente el instrumento quedó conformado por un total de 31 ítem, de los cuales los 7 primeros describían a la muestra participante y el resto indicaba el valor otorgado a las 8 herramientas Web 2.0 seleccionadas dentro de los entornos inclusivos

Realizada la prueba alfa de Cronbach para determinar la fiabilidad del instrumento encontramos que este presenta una valoración de 0.885 . Atendiendo a las tres dimensiones establecidas, la fiabilidad de cada una de ellas es 0.795, 0.809 y 0.811, respectivamente. Como vemos el instrumento presenta una fiabilidad alta (Mateo, 2004)

\section{RESULTADOS}

DIMENSIÓN 1: Valoración de algunas herramientas Web 2.0 en entornos inclusivos atendiendo a la presencia 
La tabla 3. muestra los resultados en relación a la valoración respecto de la presencia que realiza los participantes de la investigación de las herramientas Web 2.0, redes sociales, blog y edublog, foros, mensajería instantánea o chat, Webquest, Wiki, Youtube y procesadores de texto, que venimos estudiando a través de este documento.

Tabla 3. Valoración de algunas herramientas Web 2.0 en entornos inclusivos atendiendo a la presencia

\begin{tabular}{lcc}
\cline { 2 - 3 } & Media & $\mathrm{S}$ \\
\hline Valoración respecto a la presencia de los procesadores & 4.18 & 0.809 \\
de textos & & \\
Valoración respecto a la presencia de las redes sociales & 3.73 & 0.957 \\
Valoración respecto a la presencia de la mensajería & 3.54 & 1.071 \\
instantánea & & \\
Valoración respecto a la presencia de Youtube & 3.46 & 1.058 \\
Valoración respecto a la presencia de los foros & 3.40 & 1.039 \\
Valoración respecto a la presencia de los blog y & 3.35 & 1.078 \\
edublog & & \\
Valoración respecto a la presencia de la Wiki & 3.08 & 1.191 \\
Valoración respecto a la presencia de la Webquest & 3.03 & 1.142 \\
\hline
\end{tabular}

Como podemos observar en la tabla 6 , los estudiantes participantes en este estudio tienen una mejor valoración de las herramientas Web 2.0, procesadores de textos $(\bar{x}=4.18)$, redes sociales $(\bar{x}=3.73)$ y mensajería instantánea $(\bar{x}=3.54)$ para reforzar la presencia como estrategia que desarrolla procesos educativos inclusivos. En cambio, valora en menor medida la herramienta Youtube $(\bar{x}=3.46)$, los foros $(\bar{x}=3.40), \operatorname{los}$ blog y edublogs $(\bar{x}=3.35)$, la Wiki $(\bar{x}=3.08)$ y la Webquest $(\bar{x}=3.03)$.

En un segundo momento, hemos tratado de establecer diferencias estadísticamente significativas atendiendo al sexo del alumnado, aplicando una prueba $\mathrm{t}$ de Student (n.s.=0.05) cuyos resultados han revelado:

- Las mujeres valoran más positivamente los foros para reforzar. la presencia como estrategia para el desarrollo de los entornos inclusivos en comparación a los hombres $(\mathrm{t}=-2.375 \mathrm{y} \mathrm{p}=0.018)$

- La mensajería instantánea es mejor valorada por las mujeres como herramienta de fomento de la presencia en comparación con el otro sexo $(\mathrm{t}=-$ 2.896 y $\mathrm{p}=0.004)$.

- Además, la herramienta Youtube es mejor valorada por las mujeres que por los hombres para fomentar la presencia como estrategia para el desarrollo de los entornos inclusivos ( $\mathrm{t}=-2.241 \mathrm{y} \mathrm{p}=0.026)$.

Por último, el resto de las herramientas web 2.0 de este estudio, redes sociales, blog y edublog, Webquest, Wiki y procesadores de textos no revelan diferencias estadísticamente significativas en relación al sexo para esta dimensión.

Asimismo, debido a la procedencia dispar de este grupo de estudiantes en referencia a su titulación formativa, hemos realizado un Análisis de Varianza (n.s.=0.05) obteniendo los siguientes valores representativos (ver tabla 4). 
Tabla 4. Anova de la valoración de algunas herramientas Web 2.0 en entornos inclusivos atendiendo a la presencia en función de la titulación

\begin{tabular}{|c|c|c|c|c|c|}
\hline $\begin{array}{l}\text { Valoración } \\
\text { presencia de } \\
\text { algunas } \\
\text { herramientas Web } \\
2.0\end{array}$ & Titulación & $\mathrm{N}$ & Media & $\mathrm{S}$ & $\begin{array}{l}\text { F y p de los grupos } \\
\text { favorables }\end{array}$ \\
\hline \multirow[t]{5}{*}{ Redes Sociales } & $\begin{array}{l}\text { M. E. Primaria } \\
\text { G: }\end{array}$ & 65 & 3.89 & 0.868 & $\begin{array}{l}\mathrm{F}=3.151 \mathrm{y} \mathrm{p}=0.015 \\
\text { favorable }\end{array}$ \\
\hline & M. E. Física & 54 & 3.35 & 1.119 & Educación Primaria \\
\hline & M. L. Extranjera & 65 & 3.89 & 0.886 & y Lengua Extranjera \\
\hline & M. E. Musical & 43 & 3.77 & 0.841 & \\
\hline & M. N.E.E. & 77 & 3.71 & 0.971 & \\
\hline \multirow[t]{5}{*}{ Blog y edublogs } & $\begin{array}{l}\text { M. E. Primaria } \\
\text { G: }\end{array}$ & 65 & 3.65 & 1.110 & $\begin{array}{l}F=10.155 \\
p=<0.001\end{array}$ \\
\hline & M. E. Física & 54 & 2.83 & 1.042 & favorable a Lengua \\
\hline & M. L. Extranjera & 65 & 3.77 & 0.844 & Extranjera \\
\hline & M. E. Musical & 43 & 3.53 & 0.882 & \\
\hline & M. N.E.E. & 74 & 3.00 & 1.123 & \\
\hline \multirow[t]{5}{*}{ Foros } & $\begin{array}{l}\text { M. E. Primaria } \\
\text { G: }\end{array}$ & 65 & 3.86 & 0.899 & $\begin{array}{l}\mathrm{F}=6.975 \text { y } \mathrm{p}=<0.001 \\
\text { favorable }\end{array}$ \\
\hline & M. E. Física & 54 & 2.93 & 1.096 & Educación Primaria \\
\hline & M. L. Extranjera & 65 & 3.48 & 0.903 & \\
\hline & M. E. Musical & 43 & 3.42 & 0.879 & \\
\hline & M. N.E.E. & 77 & 3.26 & 1.140 & \\
\hline \multirow[t]{5}{*}{$\begin{array}{l}\text { Mensajería } \\
\text { instantánea }\end{array}$} & $\begin{array}{l}\text { M. E. Primaria } \\
\text { G: }\end{array}$ & 65 & 3.80 & 0.939 & $\begin{array}{l}\mathrm{F}=6.328 \text { y } \mathrm{p}=<0.001 \\
\text { favorable }\end{array}$ \\
\hline & M. E. Física & 54 & 3.02 & 1.189 & Educación Primaria \\
\hline & M. L. Extranjera & 65 & 3.77 & 0.897 & \\
\hline & M. E. Musical & 43 & 3.26 & 1.115 & \\
\hline & M. N.E.E. & 77 & 3.66 & 1.059 & \\
\hline \multirow[t]{5}{*}{ Webquest } & $\begin{array}{l}\text { M. E. Primaria } \\
\text { G: }\end{array}$ & 65 & 3.38 & 1.114 & $\begin{array}{l}\mathrm{F}=2.931 \mathrm{y} \mathrm{p}=0.021 \\
\text { favorable }\end{array}$ \\
\hline & M. E. Física & 54 & 2.76 & 1.115 & Educación Primaria. \\
\hline & M. L. Extranjera & 65 & 2.88 & 1.111 & \\
\hline & M. E. Musical & 43 & 2.91 & 1.065 & \\
\hline & M. N.E.E. & 75 & 3.12 & 1.196 & \\
\hline \multirow[t]{5}{*}{ Wiki } & $\begin{array}{l}\text { M. E. Primaria } \\
\text { G: }\end{array}$ & 65 & 3.52 & 1.105 & $\begin{array}{l}\mathrm{F}=5.016 \text { y } \mathrm{p}=0.001 \\
\text { favorable }\end{array}$ \\
\hline & M. E. Física & 54 & 2.61 & 1.172 & Educación Primaria \\
\hline & M. L. Extranjera & 65 & 2.98 & 1.139 & \\
\hline & M. E. Musical & 43 & 2.93 & 1.242 & \\
\hline & M. N.E.E. & 75 & 3.19 & 1.171 & \\
\hline \multirow[t]{5}{*}{ Youtube } & $\begin{array}{l}\text { M. E. Primaria } \\
\text { G: }\end{array}$ & 65 & 3.48 & 0.954 & $\begin{array}{l}\mathrm{F}=5.424 \text { y } \mathrm{p}=<0.001 \\
\text { favorable }\end{array}$ \\
\hline & M. E. Física & 54 & 2.91 & 1.086 & Educación Especial \\
\hline & M. L. Extranjera & 65 & 3.46 & 0.969 & \\
\hline & M. E. Musical & 43 & 3.67 & 1.149 & \\
\hline & M. N.E.E. & 77 & 3.70 & 1.027 & \\
\hline
\end{tabular}

El alumnado de las menciones de maestro en Educación Primaria Generalistay Lengua Extranjera realizan una valoración más favorable de la herramienta Web 2.0, redes sociales como refuerzo de la presencia en los entornos inclusivos, que el resto de los alumnos y las alumnas de las titulaciones de Educación Física, Educación Musical y Necesidades Educativas Específicas ( $\mathrm{F}=3.151$ y p=0.015). 
Las herramientas Web 2.0, blog y edublogs, es valorada más favorablemente por los alumnos y las alumnas de la titulación de Lengua Extranjera, en entornos inclusivos atendiendo a la presencia, en comparación con el alumnado de las restantes menciones $(\mathrm{F}=10.155$ y $\mathrm{p}=<0.001)$.

Los alumnos y las alumnas procedentes de la titulación de Educación Primaria Generalista valoran más positivamente los foros en entornos inclusivos atendiendo a la presencia en comparación con el alumnado de las otras titulaciones $(\mathrm{F}=6.975 \mathrm{y}$ $\mathrm{p}=<0.001)$.

La herramienta Web 2.0, mensajería instantánea, es valorada más favorablemente por los estudiantes de la titulación de Educación Primaria Generalista, en entornos inclusivos atendiendo a la presencia, en comparación con los alumnos y las alumnas de las restantes titulaciones ( $\mathrm{F}=6.328 \mathrm{y} \mathrm{p}=<0.001)$.

La Webquest, herramienta Web 2.0, es mejor valorada en entornos inclusivos atendiendo a la presencia por el alumnado de la titulación de maestro de Educación Primaria Generalista en comparación con los alumnos y las alumnas de las titulaciones de Educación Física, Lengua Extranjera, Educación Musical y Necesidades Educativas Específicas $(\mathrm{F}=2.931$ y $\mathrm{p}=0.021)$.

Los estudiantes de la titulación de maestro en Educación Primaria realiza una valoración más favorable de la herramienta Web 2.0, Wiki, en entornos inclusivos atendiendo a la presencia que el resto de los alumnos y las alumnas de las otras titulaciones $(\mathrm{F}=5.016$ y $\mathrm{p}=0.001)$.

La herramienta Web 2.0, Youtube, es más valorada como refuerzo de la presencia en los entornos inclusivos, por los alumnos y las alumnas de la titulación de Necesidades Educativas Específicas en comparación con los estudiantes de las restantes titulaciones $(\mathrm{F}=5.424$ y $\mathrm{p}=<0.001)$.

La valoración de los procesadores de texto en entornos inclusivos atendiendo a la presencia no es una herramienta Web 2.0 con carácter significativo en función de las titulaciones del alumnado participante en este estudio.

Además, se ha procedido a realizar una prueba de comparaciones múltiples post hoc para controlar la tasa de error para el conjunto total de comparaciones, denominado prueba de Scheffé, la cual ha confirmado las diferencias más significativas que hemos expuesto.

Por último, hemos considerado analizar si la forma de acceso a Internet es significativa en esta dimensión, por esta razón hemos procedido a realizar un Análisis de Varianza (n.s.=0.05), el cual ha revelado que esta variable no es significativa para la dimensión valoración de algunas herramientas Web 2.0 en entornos inclusivos atendiendo a la presencia.

Dimensión 2. Valoración de algunas herramientas web 2.0 en entornos inclusivos atendiendo a la participación

Como se observa en la tabla 5 , se muestran los resultados en relación a la valoración respecto de la participación que realiza los participantes de la investigación de las herramientas Web 2.0, redes sociales, blog y edublog, foros, mensajería instantánea o chat, Webquest, Wiki, Youtube y procesadores de texto, que venimos estudiando a través de este documento. 
Tabla 5. Valoración del alumnado respecto a la participación de las herramientas Web 2.0.

\begin{tabular}{lccc}
\cline { 2 - 4 } & Media & $\mathrm{s}$ & $\mathrm{N}$ \\
\hline Valoración respecto a la participación de los procesadores & 4.19 & 0.892 & 304 \\
de textos & & & \\
Valoración respecto a la participación de las redes sociales & 3.88 & 0.957 & 304 \\
Valoración respecto a la participación de Youtube & 3.60 & 1.039 & 304 \\
$\quad \begin{array}{l}\text { Valoración respecto a la participación de la mensajería } \\
\text { instantánea }\end{array}$ & 3.67 & 1.046 & 304 \\
Valoración respecto a la participación de los foros & & \\
$\quad$ Valoración respecto a la participación de los blog y & 3.40 & 1.072 & 304 \\
edublog & & & 303 \\
Valoración respecto a la participación de la Wiki & 3.21 & 1.204 & 304 \\
Valoración respecto a la participación de la Webquest & 3.05 & 1.155 & 302 \\
\hline
\end{tabular}

La tabla 6 muestra que los estudiantes participantes en este estudio valoran más positivamente los procesadores de textos $(\bar{x}=4.19)$, las redes sociales $(\bar{x}=3.88)$, Youtube $(\bar{x}=3.60)$, la mensajería instantánea $(\bar{x}=3.67)$ y los foros $(\bar{x}=3.52)$ como herramientas Web 2.0 que refuerzan la participación, estrategia de desarrollo de los procesos educativos inclusivos. En cambio realizan una menor valoración de los blog y edublogs, de la Wiki y de la Webquest ( $\bar{x}=3.40 ; 3.21$ y 3.05 respectivamente).

Considerando esta misma dimensión "Valoración de algunas herramientas Web 2.0 en entornos inclusivos atendiendo a la participación" hemos tratado de establecer diferencias estadísticamente significativas atendiendo al sexo del alumnado, aplicando una prueba $t$ de Student (n.s.=0.05), la cual ha revelado los siguientes resultados más significativos.

Tabla 6. T Student de la valoración de algunas herramientas Web 2.0 atendiendo a la participación en función del sexo

\begin{tabular}{|c|c|c|c|c|c|}
\hline $\begin{array}{c}\text { Valoración } \\
\text { participación de algunas } \\
\text { herramientas Web } 2.0\end{array}$ & Sexo & $\mathrm{N}$ & Media & $\mathrm{S}$ & $\begin{array}{l}\text { t y p de los grupos } \\
\text { favorables }\end{array}$ \\
\hline \multirow[t]{2}{*}{ Redes sociales } & Hombre & 89 & 3.63 & 1.038 & $\mathrm{t}=.-2.830 . \mathrm{y} \cdot \mathrm{p}=0.015$ \\
\hline & Mujer & 215 & 3.99 & 0.904 & A favor de las mujeres \\
\hline \multirow[t]{2}{*}{ Foros } & Hombre & 89 & 3.28 & 1.044 & $\mathrm{t}=-2.487 . \quad \mathrm{y} \cdot \mathrm{p}=0.013$ \\
\hline & Mujer & 215 & 3.61 & 1.070 & A favor de las mujeres \\
\hline \multirow[t]{2}{*}{ Mensajería instantánea } & Hombre & 89 & 3.35 & 1.129 & $\mathrm{t}=-3.293 . \mathrm{y} \cdot \mathrm{p}=0.001$ \\
\hline & Mujer & 215 & 3.80 & 0.982 & A favor de las mujeres \\
\hline \multirow[t]{2}{*}{ Wiki } & Hombre & 89 & 2.94 & 1.152 & $\mathrm{t}=-2.538 . \quad \mathrm{y} \cdot \mathrm{p}=0.012$ \\
\hline & Mujer & 215 & 3.33 & 1.210 & A favor de las mujeres \\
\hline \multirow[t]{2}{*}{ Youtube } & Hombre & 89 & 3.30 & 0.958 & $\mathrm{t}=-3.200 . \quad \mathrm{y} \cdot \mathrm{p}=0.002$ \\
\hline & Mujer & 215 & 3.72 & 1.049 & A favor de las mujeres \\
\hline
\end{tabular}

En este sentido, en relación a la dimensión "Valoración de algunas herramientas Web 2.0 en entornos inclusivos atendiendo a la participación" hemos tratado de establecer diferencias estadísticamente significativas atendiendo al sexo del alumnado, aplicando una prueba $\mathrm{t}$ de Student (n.s.=0.05) cuyos resultados han revelado: 
- Las redes sociales son mejor valoradas como herramientas Web 2.0 que refuerzan la participación en entornos inclusivos por las mujeres que por el otro sexo $(\mathrm{t}=-2.830$ y $\mathrm{p}=0.005)$.

- Las mujeres valoran más positivamente los foros para reforzar. la participación como estrategia para el desarrollo de los entornos inclusivos en comparación a los hombres ( $\mathrm{t}=-2.487$ y $\mathrm{p}=0.013)$.

- La mensajería instantánea es mejor valorada por las mujeres como herramienta de fomento de la participación ( $\mathrm{t}=-3.293$ y $\mathrm{p}=0.001)$.

- Las mujeres valoran más positivamente la Wiki para reforzar. la participación como estrategia para el desarrollo de los entornos inclusivos en comparación a los hombres $(\mathrm{t}=-2.538$ y $\mathrm{p}=0.012)$.

- Además, la herramienta Youtube es mejor valorada por las mujeres que por los hombres para fomentar la participación en los entornos inclusivos ( $\mathrm{t}=-$ 3.200 y $\mathrm{p}=0.002)$.

Por último, el resto de las herramientas Web 2.0 de este estudio, blog y edublog, Webquest, y procesadores de textos no revelan diferencias estadísticamente significativas en relación al sexo para esta dimensión.

De la misma forma, debido a la adscripción de este grupo de alumnos y las alumnas a diferentes titulaciones formativas, hemos considerado necesario realizar un Análisis de Varianza (n.s.=0.05) obteniendo los siguientes valores representativos que se muestran en la tabla 7.

Tabla 7. Anova de la valoración del alumnado respecto a la participación de algunas herramientas Web 2.0 en función de la titulación

\begin{tabular}{|c|c|c|c|c|c|}
\hline $\begin{array}{c}\text { Valoración } \\
\text { participación de } \\
\text { algunas herramientas } \\
\text { Web } 2.0\end{array}$ & Titulación & $\mathrm{N}$ & Media & $\mathrm{S}$ & $\begin{array}{l}\text { F y p de los grupos } \\
\text { favorables }\end{array}$ \\
\hline \multirow[t]{5}{*}{ Redes Sociales } & $\begin{array}{lll}\text { M. } & \text { E. Primaria } \\
\text { G: } & \end{array}$ & 65 & 4.02 & 0.927 & \multirow{5}{*}{$\begin{array}{l}\mathrm{F}=4.434 \mathrm{y} \mathrm{p}=0.002 \\
\text { favorable a Lengua } \\
\text { Extranjera }\end{array}$} \\
\hline & M. E. Física & 54 & 3.41 & 1.125 & \\
\hline & M. L. Extranjera & 65 & 4.05 & 0.759 & \\
\hline & M. E. Musical & 43 & 3.88 & 0.793 & \\
\hline & M. N.E.E. & 77 & 3.96 & 1.006 & \\
\hline \multirow[t]{5}{*}{ Blog y edublogs } & $\begin{array}{l}\text { M. E. Primaria } \\
\text { G: }\end{array}$ & 65 & 3.71 & 1.086 & \multirow{5}{*}{$\begin{array}{l}\mathrm{F}=11.347 \\
\mathrm{p}=<0.001 \\
\text { favorable a Lengua } \\
\text { Extranjera }\end{array}$} \\
\hline & M. E. Física & 54 & 2.91 & 1.137 & \\
\hline & M. L. Extranjera & 65 & 3.83 & 0.840 & \\
\hline & M. E. Musical & 43 & 3.65 & 0.948 & \\
\hline & M. N.E.E. & 76 & 2.96 & 1.113 & \\
\hline \multirow[t]{5}{*}{ Foros } & $\begin{array}{lll}\text { M. } & \text { E. Primaria } \\
\text { G: } & \end{array}$ & 65 & 3.92 & 1.020 & \multirow{5}{*}{$\begin{array}{l}\mathrm{F}=7.785 \text { y } \mathrm{p}=<0.001 \\
\text { favorable } \\
\text { Educación Primaric } \\
\text { Generalista }\end{array}$} \\
\hline & M. E. Física & 54 & 2.98 & 1.000 & \\
\hline & M. L. Extranjera & 65 & 3.77 & 0.825 & \\
\hline & M. E. Musical & 43 & 3.51 & 0.985 & \\
\hline & M. N.E.E. & 77 & 3.34 & 1.210 & \\
\hline
\end{tabular}




\begin{tabular}{|c|c|c|c|c|c|}
\hline \multirow[t]{5}{*}{$\begin{array}{l}\text { Mensajería } \\
\text { instantánea }\end{array}$} & $\begin{array}{lll}\text { M. E. Primaria } \\
\text { G: }\end{array}$ & 65 & 3.92 & 1.005 & \multirow{5}{*}{$\begin{array}{l}\mathrm{F}=7.983 \text { y } \mathrm{p}=<0.001 \\
\text { favorable a Lengua } \\
\text { Extranjera }\end{array}$} \\
\hline & M. E. Física & 54 & 3.04 & 1.165 & \\
\hline & M. L. Extranjera & 65 & 3.97 & 0.728 & \\
\hline & M. E. Musical & 43 & 3.58 & 1.074 & \\
\hline & M. N.E.E. & 77 & 3.69 & 1.029 & \\
\hline \multirow[t]{5}{*}{ Webquest } & $\begin{array}{lll}\text { M. } & \text { E. Primaria } \\
\text { G: } & & \end{array}$ & 65 & 3.43 & 1.104 & \multirow{5}{*}{$\begin{array}{l}\mathrm{F}=4.557 \text { y } \mathrm{p}=0.001 \\
\text { favorable } \\
\text { Educación Primaria } \\
\text { Generalista }\end{array}$} \\
\hline & M. E. Física & 54 & 2.56 & 1.076 & \\
\hline & M. L. Extranjera & 65 & 3.12 & 1.038 & \\
\hline & M. E. Musical & 43 & 2.98 & 1.205 & \\
\hline & M. N.E.E. & 75 & 3.04 & 1.213 & \\
\hline \multirow[t]{5}{*}{ Wiki } & $\begin{array}{l}\text { M. E. Primaria } \\
\text { G: }\end{array}$ & 65 & 3.78 & 1.023 & \multirow{5}{*}{$\begin{array}{l}\mathrm{F}=9.464 \text { y } \mathrm{p}=<0.001 \\
\text { favorable } \\
\text { Educación Primaria } \\
\text { Generalista }\end{array}$} \\
\hline & M. E. Física & 54 & 2.69 & 1.146 & \\
\hline & M. L. Extranjera & 65 & 3.49 & 1.033 & \\
\hline & M. E. Musical & 43 & 2.81 & 1.200 & \\
\hline & M. N.E.E. & 77 & 3.09 & 1.279 & \\
\hline \multirow[t]{5}{*}{ Youtube } & $\begin{array}{lll}\text { M. } & \text { E. Primaria } \\
\text { G: } & & \end{array}$ & 65 & 3.72 & 0.944 & \multirow{5}{*}{$\begin{array}{l}\mathrm{F}=5.895 \text { y } \mathrm{p}=<0.001 \\
\text { favorable a Lengua } \\
\text { Extranjera }\end{array}$} \\
\hline & M. E. Física & 54 & 3.00 & 0.991 & \\
\hline & M. L. Extranjera & 65 & 3.80 & 1.003 & \\
\hline & M. E. Musical & 43 & 3.65 & 0.842 & \\
\hline & M. N.E.E. & 77 & 3.70 & 1.148 & \\
\hline
\end{tabular}

Los estudiantes de la titulación de maestro en Lengua Extranjera realizan una valoración más favorable de la herramienta Web 2.0, redes sociales como refuerzo de la participación en los entornos inclusivos, que el resto de los alumnos y las alumnas de las titulaciones de Educación Primaria Generalista, Educación Física, Educación Musical y Necesidades Educativas Específicas ( $\mathrm{F}=4.434$ y p=0.002).

Las herramientas Web 2.0, blog y edublogs, son valoradas más favorablemente por los alumnos y las alumnas de la titulación de Lengua Extranjera, en entornos inclusivos atendiendo a la participación, en comparación con el alumnado de las restantes titulaciones $(\mathrm{F}=11.347 \mathrm{y} \mathrm{p}=<0.001)$.

Los estudiantes procedentes de la titulación de Educación Primaria Generalista valoran más positivamente los foros en entornos inclusivos atendiendo a la participación en comparación con el resto de estudiantes de las otras titulaciones $(\mathrm{F}=7.785$ y $\mathrm{p}=<0.001)$.

La herramienta Web 2.0, mensajería instantánea, es valorada más favorablemente por los estudiantes de la titulación de Lengua Extranjera, en entornos inclusivos atendiendo a la participación, en comparación con el alumnado de las restantes titulaciones $(\mathrm{F}=7.983$ y $\mathrm{p}=<0.001)$.

La Webquest, es mejor valorada, como herramienta Web 2.0, para el refuerzo de la participación en los procesos educativos inclusivos, por el alumnado de la titulación de maestro de Educación Primaria Genralista en comparación con los alumnos y las alumnas de las titulaciones de Educación Física, Lengua Extranjera, Educación Musical y Necesidades Educativas Específicas ( $\mathrm{F}=4.557$ y p=0.001).

Los estudiantes de la titulación de maestro en Educación Primaria Generalista realiza una valoración más favorable de la herramienta Web 2.0, Wiki, en entornos inclusivos atendiendo a la participación que el resto del alumnado de las otras titulaciones $(\mathrm{F}=9.464 \mathrm{y} \mathrm{p}=<0.001)$. 
La herramienta Web 2.0, Youtube, es más valorada como refuerzo de la participación en los entornos inclusivos, por el alumnado de la titulación de Lengua Extranjera. en comparación con los estudiantes de las restantes titulaciones $(\mathrm{F}=5.895 \mathrm{y}$ $\mathrm{p}=<0.001)$.

La valoración de los procesadores de texto en entornos inclusivos atendiendo a la participación no es una herramienta Web 2.0 con carácter significativo en función de las titulaciones del alumnado participante en este estudio.

Además, se ha procedido a realizar una prueba de comparaciones múltiples post hoc para controlar la tasa de error para el conjunto total de comparaciones, denominado prueba de Scheffé, la cual ha confirmado las diferencias más significativas que hemos expuesto.

Por último, hemos considerado analizar si la forma de acceso a Internet es significativa en esta dimensión, por esta razón hemos procedido a realizar un Análisis de Varianza (n.s. $=0.05)$, el cual ha revelado:

- La valoración de las redes sociales como herramienta Web 2.0 que refuerza la participación en los procesos educativos inclusivos es más favorable en los alumnos y las alumnas que acceden a Internet a través del móvil en comparación con el resto de estudiantes que realizan otra forma de acceso $(\mathrm{F}=2.456$ у $\mathrm{p}=0.034)$.

- Los estudiantes que acceden a Internet a través de todas las modalidades de forma conjunta valoran más positivamente la mensajería instantánea en los entornos inclusivos para reforzar la participación $(\mathrm{F}=2.268$ y p=0.048).

- Los procesadores de textos son valorados mayormente como herramienta Web 2.0 que refuerza la participación en entornos inclusivos por los estudiantes que acceden a Internet a través del móvil $(\mathrm{F}=2.441$ y p=0.034).

El resto de herramientas de este estudio no han reflejado resultados significativos en función del tipo de acceso a Internet para la dimensión valoración de algunas herramientas Web 2.0 en entornos inclusivos atendiendo a la participación.

Además, se ha procedido a realizar una prueba de comparaciones múltiples post hoc para controlar la tasa de error para el conjunto total de comparaciones, denominado prueba de Scheffé, la cual ha confirmado las diferencias más significativas que hemos expuesto.

Dimensión 3. Valoración de algunas herramientas Web 2.0 en entornos inclusivos atendiendo al progreso

La tabla 8 muestra los resultados en relación a la valoración respecto del progreso que realiza los participantes de la investigación de las herramientas web 2.0, redes sociales, blog y edublog, foros, mensajería instantánea o chat, Webquest, Wiki, Youtube y procesadores de texto, que venimos estudiando a través de este documento. 
Tabla 8. Valoración del alumnado respecto a la participación de las herramientas Web 2.0.

\begin{tabular}{lccc}
\cline { 2 - 4 } & Media & $\mathrm{s}$ & $\mathrm{N}$ \\
\hline Valoración respecto al progreso de los procesadores de & 4.27 & 0.780 & 298 \\
textos & & & \\
Valoración respecto al progreso de las redes sociales & 3.79 & 0.964 & 299 \\
$\begin{array}{l}\text { Valoración respecto al progreso de la mensajería } \\
\text { instantánea }\end{array}$ & 3.61 & 1.031 & 297 \\
Valoración respecto al progreso de Youtube & & & \\
Valoración respecto al progreso de los foros & 3.60 & 1.032 & 299 \\
Valoración respecto al progreso de los blog y edublog & 3.55 & 1.027 & 299 \\
Valoración respecto al progreso de la Wiki & 3.53 & 1.078 & 299 \\
Valoración respecto al progreso de la Webquest & 3.34 & 1.200 & 299 \\
\hline
\end{tabular}

Como podemos observar en la tabla 9 , los alumnos y las alumnas participantes en este estudio realizan una mejor valoración de los procesadores de textos $(\bar{x}=4.27)$, de las redes sociales $(\bar{x}=3.79)$, de la mensajería instantánea $(\bar{x}=3.61)$, de Youtube $(\bar{x}=3.60)$, de los foros $(\bar{x}=3.55)$ y de los blog y edublogs $(\bar{x}=3.53)$ como herramientas Web 2.0 que refuerzan el progreso en los entornos inclusivos. En cambio poseen un menor conocimiento de la Wiki $(\bar{x}=3.34)$ y de la Webquest $(\bar{x}=3.21)$ a este respecto.

En este sentido, en relación a la dimensión "Valoración de algunas herramientas Web 2.0 en entornos inclusivos atendiendo al progreso" hemos tratado de establecer diferencias estadísticamente significativas atendiendo al sexo del alumnado, aplicando una prueba $\mathrm{t}$ de Student (n.s.=0.05) cuyos resultados más significativos se muestran en la tabla 0 .

Tabla 9. T Student de la valoración de algunas herramientas Web 2.0 atendiendo al progreso en función del sexo

\begin{tabular}{ccrccc}
\hline $\begin{array}{c}\text { Valoración progreso de } \\
\text { algunas herramientas } \\
\text { Web 2.0 }\end{array}$ & Sexo & $\mathrm{N}$ & Media & $\mathrm{S}$ & $\begin{array}{c}\mathrm{t} \text { y p de los grupos } \\
\text { favorables }\end{array}$ \\
\hline Redes sociales & Hombre & 89 & 3.55 & 0.989 & $\mathrm{t}=-2.860 \mathrm{y} \cdot \mathrm{p}=0.005$ \\
Foros & Mujer & 210 & 3.90 & 0.938 & A favor de las mujeres \\
& Hombre & 89 & 3.24 & 1.128 & $\mathrm{t}=-3.261 \mathrm{y} \cdot \mathrm{p}=0.001$ \\
Mensajería instantánea & Mujer & 210 & 3.68 & 0.953 & A favor de las mujeres \\
& Hombre & 89 & 3.24 & 1.098 & $\mathrm{t}=-4.238 \mathrm{y} \cdot \mathrm{p}=0.000$ \\
Youtube & Mujer & 208 & 3.77 & 0.959 & A favor de las mujeres \\
& Hombre & 89 & 3.42 & 1.020 & $\mathrm{t}=-2.042 \mathrm{y} \cdot \mathrm{p}=0.042$ \\
& Mujer & 210 & 3.68 & 1.030 & A favor de las mujeres \\
\hline
\end{tabular}

La tabla 10 muestra que las mujeres realizan una mejor valoración de redes sociales $(\mathrm{t}=-2.860$ y $\mathrm{p}=0.005)$, los foros $(\mathrm{t}=-3.261$ y $\mathrm{p}=0.001)$, la mensajería instantánea $(\mathrm{t}=-4.238$ y $\mathrm{p}=0.000)$ y el Youtube $(\mathrm{t}=-2.042$ y $\mathrm{p}=0.042)$ como herramientas Web 2.0 que refuerzan el progreso para desarrollar procesos educativos inclusivos, en comparación con los hombres.

La valoración de las restantes herramientas Web 2.0 (blog y edublogs, Webquest, Wiki y procesadores de texto) atendiendo al progreso en entornos inclusivos no revela resultados estadísticamente significativos en función del sexo.

De la misma forma, dada la procedencia dispar de este grupo de estudiantes a diferentes titulaciones formativas, hemos considerado necesario realizar un Análisis de 
Varianza (n.s. $=0.05)$ obteniendo los siguientes valores representativos que se muestran en la tabla 10.

Tabla 10. Anova de la valoración del alumnado respecto al progreso de algunas herramientas Web 2.0 en función de la titulación

\begin{tabular}{|c|c|c|c|c|c|}
\hline $\begin{array}{c}\text { Valoración progreso } \\
\text { de algunas } \\
\text { herramientas Web } \\
2.0\end{array}$ & Titulación & $\mathrm{N}$ & Media & $S$ & $\begin{array}{c}\text { F y p de los } \\
\text { grupos favorables }\end{array}$ \\
\hline \multirow{6}{*}{ Redes Sociales } & E. Primaria & 65 & 3.95 & 0.874 & $\mathrm{~F}=3.857$ \\
\hline & E. Física & 54 & 3.35 & 1.012 & $\mathrm{p}=0.005$ \\
\hline & L. & 65 & 3.94 & 0.840 & favorable \\
\hline & Extranjera & & & & Educación \\
\hline & E. Musical & 42 & 3.88 & 0.772 & Primaria \\
\hline & E. Especial & 75 & 3.80 & 1.115 & Generalista \\
\hline \multirow[t]{6}{*}{ Blog y edublogs } & E. Primaria & 65 & 3.89 & 0.831 & $F=7.789$ \\
\hline & E. Física & 54 & 3.11 & 1.022 & $\mathrm{p}=<0.001$ \\
\hline & L. & 63 & 3.81 & 0.913 & favorable \\
\hline & Extranjera & & & & Educación \\
\hline & E. Musical & 42 & 3.71 & 0.995 & Primaria \\
\hline & E. Especial & 75 & 3.19 & 1.281 & Generalista \\
\hline \multirow[t]{6}{*}{ Foros } & E. Primaria & 65 & 3.92 & 0.835 & $\mathrm{~F}=7.352$ \\
\hline & E. Física & 54 & 3.02 & 1.107 & $\mathrm{p}=<0.001$ \\
\hline & L. & 63 & 3.78 & 0.812 & favorable \\
\hline & Extranjera & & & & Educación \\
\hline & E. Musical & 42 & 3.43 & 0.941 & Primaria \\
\hline & E. Especial & 75 & 3.48 & 1.155 & Generalista \\
\hline \multirow{6}{*}{$\begin{array}{l}\text { Mensajería } \\
\text { instantánea }\end{array}$} & E. Primaria & 65 & 3.89 & 0.850 & $\mathrm{~F}=6.664$ \\
\hline & E. Física & 54 & 3.04 & 1.132 & $\mathrm{p}=<0.001$ \\
\hline & L. & 63 & 3.83 & 0.871 & favorable \\
\hline & Extranjera & & & & Educación \\
\hline & E. Musical & 42 & 3.52 & 1.018 & Primaria \\
\hline & E. Especial & 73 & 3.66 & 1.083 & Generalista \\
\hline \multirow[t]{6}{*}{ Webquest } & E. Primaria & 65 & 3.51 & 1.017 & $\mathrm{~F}=3.920$ \\
\hline & E. Física & 54 & 2.93 & 1.163 & $\mathrm{p}=0.004$ favorable \\
\hline & L. & 63 & 3.33 & 0.950 & a Educación \\
\hline & Extranjera & & & & Primaria \\
\hline & E. Musical & 42 & 2.76 & 1.226 & Generalista \\
\hline & E. Especial & 74 & 3.30 & 1.279 & \\
\hline \multirow[t]{6}{*}{ Wiki } & E. Primaria & 65 & 3.77 & 1.012 & $\mathrm{~F}=5.625$ \\
\hline & E. Física & 54 & 2.94 & 1.220 & $\mathrm{p}=<0.001$ \\
\hline & L. & 63 & 3.52 & 0.931 & favorable \\
\hline & Extranjera & & & & Educación \\
\hline & E. Musical & 42 & 2.90 & 1.394 & Primaria \\
\hline & E. Especial & 75 & 3.35 & 1.279 & Generalista \\
\hline \multirow[t]{6}{*}{ Youtube } & E. Primaria & 65 & 3.75 & 0.902 & $\mathrm{~F}=3.671$ \\
\hline & E. Física & 54 & 3.13 & 1.100 & $\mathrm{p}=0.006$ \\
\hline & L. & 63 & 3.70 & 0.961 & favorable \\
\hline & Extranjera & & & & Educación \\
\hline & E. Musical & 42 & 3.74 & 0.828 & Primaria \\
\hline & E. Especial & 75 & 3.65 & 1.168 & Generalista \\
\hline
\end{tabular}


Los estudiantes de la titulación de maestro en Educación Primaria Generalista realizan una valoración más favorable de la herramienta Web 2.0, redes sociales como refuerzo del progreso en los entornos inclusivos, que el resto del alumnado de las titulaciones de Educación Primaria Generalista, Educación Física, Educación Musical y Necesidades Educativas Específicas ( $\mathrm{F}=3.857$ y $\mathrm{p}=0.005$ ).

Las herramientas Web 2.0, blog y edublogs, es valorada más favorablemente por el alumnados de la titulación de Educación Primaria, en entornos inclusivos atendiendo al progreso, en comparación con los estudiantes de las restantes titulaciones $(\mathrm{F}=7.789 \mathrm{y} \mathrm{p}=<0.001)$.

Los estudiantes procedentes de la titulación de Educación Primaria Generalista valoran más positivamente los foros en entornos inclusivos atendiendo al progreso en comparación con el resto de alumnos y las alumnas de las otras titulaciones $(\mathrm{F}=7.352$ y $\mathrm{p}=<0.001)$.

La herramienta Web 2.0, mensajería instantánea, es valorada más favorablemente por el discente de la titulación de Educación Primaria Generalista, en entornos inclusivos atendiendo al progreso, en comparación con los estudiantes de las restantes titulaciones $(\mathrm{F}=6.664 \mathrm{y} \mathrm{p}=<0.001)$.

La Webquest, es mejor valorada, como herramienta web 2.0, para el refuerzo del progreso en los procesos educativos inclusivos, por el alumnado de la titulación de maestro de Educación Primaria Generalista en comparación con el alumnado de las titulaciones de Educación Física, Lengua Extranjera, Educación Musical y Necesidades Educativas Específicas ( $\mathrm{F}=3.920$ y p=0.004).

Los estudiantes de la titulación de maestro en Educación Primaria Generalista realiza una valoración más favorable de la herramienta Web 2.0, Wiki, en entornos inclusivos atendiendo al progreso que el resto de los alumnos y las alumnas de las otras titulaciones $(\mathrm{F}=5.625 \mathrm{y} \mathrm{p}=<0.001)$.

La herramienta Web 2.0, Youtube, es más valorada como refuerzo del progreso en los entornos inclusivos, por el alumnado de la titulación de Educación Primaria Generalista en comparación con los estudiantes de las restantes titulaciones $(\mathrm{F}=3.671$ y $\mathrm{p}=0.006)$.

La valoración de los procesadores de texto en entornos inclusivos atendiendo al progreso no es una herramienta Web 2.0 con carácter significativo en función de las titulaciones de los estudiantes participantes en este estudio.

Además, se ha procedido a realizar una prueba de comparaciones múltiples post hoc para controlar la tasa de error para el conjunto total de comparaciones, denominado prueba de Scheffé, la cual ha confirmado las diferencias más significativas que hemos expuesto.

Finalmente, hemos considerado analizar si la forma de acceso a Internet es significativa en la dimensión "Valoración de algunas herramientas Web 2.0 en entornos inclusivos atendiendo al progreso", consecuentemente hemos procedido a realizar un Análisis de Varianza (n.s.=0.05), el cual ha revelado:

- La valoración de la Wiki como herramienta Web 2.0 que refuerza el progreso en los procesos educativos inclusivos es más favorable en los alumnos y las alumnas que acceden a Internet a través del móvil en comparación con el resto de estudiantes que realizan otra forma de acceso ( $F=3.499$ y $p=0.004)$. 
- Los estudiantes que acceden a Internet a través del móvil valoran más positivamente Youtube en los entornos inclusivos para reforzar el progreso $(\mathrm{F}=2.660$ y $\mathrm{p}=0.023)$.

- Los procesadores de textos son valorados mayormente, como herramienta web 2.0 que refuerza el progreso en entornos inclusivos, por los estudiantes que acceden a Internet a través de todas las modalidades de forma conjunta $(\mathrm{F}=5.134$ y $\mathrm{p}=<0.001)$.

El resto de herramientas de este estudio no han reflejado resultados significativos en función del tipo de acceso a Internet para la dimensión valoración de algunas herramientas Web 2.0 en entornos inclusivos atendiendo al progreso.

De la misma forma, se ha procedido a realizar una prueba de comparaciones múltiples post hoc para controlar la tasa de error para el conjunto total de comparaciones (prueba de Scheffé) la cual ha confirmado las diferencias más significativas que hemos expuesto.

\section{CONCLUSIONES}

El empleo de las TIC en todos los niveles educativos es significativo, tanto a nivel investigador como experiencias de innovación docente (Trujillo, Hinojo, Marín, Romero y Campos, 2015). Una de las líneas que va cobrando poco a poco un gran valor es la relación de la educación inclusiva y las tecnologías.

Como decimos los entornos inclusivos y el desarrollo de las herramientas Web 2.0 van de la mano en los últimos tiempos, sin embargo el valor que se les da a estos no es el mismo pues depende del espacio en que su empleo se lleve a cabo. Así encontramos que en entornos formales suelen ser muy valorados y apreciados, mientras que en los inclusivos los empleadores a veces muestran una mayor reticencia a su empleo bien por su desconocimiento de uso bien por no saber combinar su aplicación con la variable inclusión.

En esta línea se ha comprobado como los futuros maestros de Educación Primaria valoran la presencia de las herramientas tecnológicas en el ámbito de la educación inclusiva de manera positiva. Sin embargo es llamativo, que aquellos maestros en formación que cursan la mención de Necesidades Educativas Especiales consideren que solo Youtube tiene una valoración positiva y significativa para ser empleada en entornos inclusivos, frente a los que cursan la mención generalista del grado de Primaria.

Por otra parte, como vemos en todos los casos son las mujeres las que se posicionan más abiertas a valorar la presencia y usabilidad de las herramientas Web 2.0 en este tipo de ámbitos, por lo que cabe hacerse dos cuestiones, que darían pie a la ampliación de este estudio, de un lado ¿es la utilización por parte de los docentes que participan en las menciones la que determina las creencias hacia el valor de las herramientas Web 2.0 en las aulas o entornos inclusivos? Y de otro, ¿es la alta tasa de mujeres en esta titulación la que determina estas puntuaciones y provoca, en consecuencia un sesgo referido al género? 


\section{REFERENCIAS}

Cabero, J., \& Córdoba, M. (2009). Inclusión Educativa: inclusión digital. Revista de Educación Inclusiva, 2(1), 61-78.

Casanova, J., \& González, J. (2010). Educación inclusiva y otros conceptos afines para el desarrollo. Escuela para todos en la sociedad de la información. Revista Educação Arte e Inclusão, 1(3). Recuperado de: http://www.periodicos.udesc.br/index.php/arteinclusao/article/view/2109

Castaño, C., Maiz, I., Palacio, G. \& Villaroel, J.D. (2008). Prácticas educativas en entornos Web 2.0. Madrid: Síntesis (coord).

León, O. G. (2003). Diseños “ex post facto”. En O.G. León e I. Montero (Coords), Métodos de investigación en psicología y educación (p. 359-394). Madrid: Mc Graw Hill.

Marín, V., \& Reche, E. (2011). Desarrollo de la Competencia Digital en Educación Secundaria. En V. Marín Díaz (coord.), Cómo trabajar la competencia digital en Educación Secundaria (pp. 45-61). Sevilla: Mad, Eduforma.

Mateo, J. (2004). La investigación ex post facto. En R. Bisquerra (Coord.), Metodología de la investigación educativa (pp.45-51). Madrid: La Muralla.

Pérez, R., García, J.L., Gil, J. A., \& Galán, A. (2009). Estadística aplicada a la educación. Madrid: Pearson Educación y UNED.

Muntaner, J. J. (2010). De la integración a la inclusión: un nuevo modelo educativo. En Arnaiz, P.; Hurtado, M.D. y Soto, F. J. (Coords), 25 años de Integración Escolar en España: Tecnología e Inclusión en el ámbito educativo, laboral y comunitario. Murcia: Consejería de Educación, Formación y Empleo.

Trujillo, J. M., Hinojo, M. A., Marín, J. A., Romero, J. J., \& Campos, A. (2015). Análisis de experiencias de aprendizajes basados en proyectos: prácticas colaborativas B-Learning. EDMETIC, Revista de Educación Mediática y TIC, 4(1), $51-77$. Recuperado de http://www.uco.es/ucopress/ojs/index.php/edmetic/article/view/2899/2823 